\title{
Hippocampus and Contextual Fear Conditioning: Recent Controversies and Advances
}

\author{
Stephan G. Anagnostaras, Greg D. Gale, and \\ Michael S. Fanselow \\ Department of Psychology and Brain Research Institute, \\ University of California, Los Angeles, California
}

\begin{abstract}
Dorsal hippocampal (DH) lesions produce a severe deficit in recently, but not remotely, acquired contextual fear without impairing memory of discrete training stimuli, i.e., $\mathrm{DH}$ lesions produce an anterograde and time-limited retrograde amnesia specific to contextual memory. These data are consistent with the standard model which posits temporary involvement of the hippocampus in recent memory maintenance. However, three recent controversies apparently weaken the case for a selective mnemonic role for the hippocampus in contextual fear. First, although retrograde amnesia (from posttraining lesions) is severe, anterograde amnesia (from pretraining lesions) may be mild or nonexistent. Second, a performance, rather than mnemonic, account of contextual freezing deficits in hippocampal-lesioned animals has been offered. Third, damage to the entire hippocampus, including the ventral hippocampus, can produce a dramatic and temporally stable disruption of context and tone fear. These data are reviewed and explanations are offered as to why they do not necessarily challenge the standard model of hippocampal memory function in contextual fear. Finally, a more complete description of the hippocampus' proposed role in contextual fear is offered, along with new data supporting this view. In summary, the data support a specific mnemonic role for the DH in the acquisition and consolidation of contextual representations. Hippocampus 2001;11:8-17.

๑) 2001 Wiley-Liss, Inc.
\end{abstract}

KEY WORDS: lesion; gradient

consolidation; amnesia; configural; spatial; amygdala;

\section{INTRODUCTION}

It is widely recognized that after damage to the human hippocampal formation, an amnesic syndrome ensues. In amnesics, an anterograde amnesia (AA; an inability to form new memories) is accompanied by a retrograde amnesia (RA; a loss of memory acquired before the trauma). These memory failures are specific to declarative, as opposed to nondeclarative, memory. The extent of RA can be quite variable, but is typically temporally graded, i.e., memories acquired just prior to the lesion (recent memory) are more severely impacted than those acquired several years before (remote memory; Scoville and Milner, 1957; Squire and Alvarez, 1995; Knowlton and Fanselow, 1998; but see also Nadel and Moscovitch, 1997, 1998). In

Grant sponsor: NSF; Grant number: IBN 9723295; Grant sponsor: UCLA C.M. Kernan Dissertation Year Fellowship.

*Correspondence to: Michael S. Fanselow, Department of Psychology, University of California at Los Angeles, 1285 Franz Hall, Los Angeles, CA 90095-1563. E-mail: fanselow@ucla.edu

Accepted for publication 1 August 2000 humans, temporally graded RA is inferred through the use of retrospective memory tests, such as those examining autobiographical details, public events, and television shows (e.g., Rimpel-Clower et al., 1996; Reed and Squire, 1998; Squire, 1992).

Many studies in animals have examined the effect of hippocampal lesions made prior to training (e.g., Olton et al., 1979; Morris, 1983; Phillips and LeDoux, 1992; Kim et al., 1993), but relatively few have examined temporally graded RA after damage to the hippocampal formation (Zola-Morgan and Squire, 1990; Winocur, 1990; Kim and Fanselow, 1992; Kim et al., 1995; Bolhuis et al., 1994; Cho et al., 1993; Cho and Kesner, 1996; Wiig et al., 1996; Maren et al., 1997; Anagnostaras et al., 1999; for a summary, see Milner et al., 1998).

Aspects of hippocampal lesion-induced amnesia can be found in Pavlovian fear conditioning, in which a tone conditional stimulus (CS) is paired with a shock unconditional stimulus (US) several times in a novel context. Rats trained in this manner develop a fear of both the tone and training context, which we measure as freezing, an adaptive species-specific defense reaction (Bolles, 1970; Fanselow, 1980). Contextual fear acquisition may involve configural or spatial learning (Nadel and Willner, 1980; Sutherland and Rudy, 1989), and many lines of evidence support hippocampal involvement in contextual fear conditioning (see, for example, Maren et al., 1998; Phillips and LeDoux, 1992).

In our laboratory, we examined temporally graded RA of contextual fear in three studies. Kim and Fanselow (1992) gave rats tone-shock pairings either 1, 7, 14, or 28 days before the animals received an electrolytic lesion of the dorsal hippocampus (DH) or sham surgery. In this study, $\mathrm{DH}$ lesions made 1 day after training produced a near-complete deficit in contextual fear, while sparing tone freezing. In contrast, lesions made 28 days after training failed to produce a reliable deficit. Hippocampal lesions produced a selective and time-limited RA of contextual fear (Fig. 1A). This led us to suggest that the hippocampus plays a temporary role in the formation of some aspects of contextual fear memory, which must become independent of the hippocampus over some time period after training. 

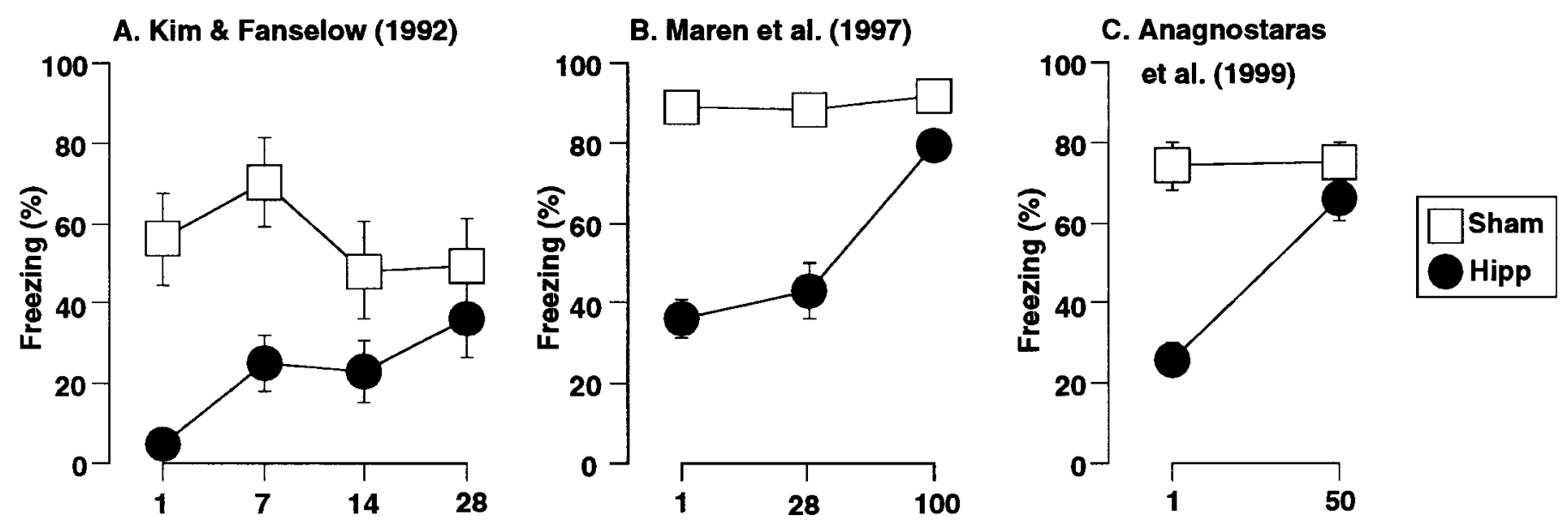

\section{Training-to-lesion interval (Days) \\ (Recent ->Remote Memory)}

FIGURE 1. Summary of studies examining RA of contextual fear from our laboratory. A: From Kim and Fanselow (1992). Rats were given 10 tone-shock pairings and then given electrolytic lesions of the DH 1, 7, 14, or 28 days after training. After recovery, contextual freezing (\% time, mean \pm SEM) was assessed. A substantial deficit was observed when lesions were made 1 day, but not 28 days, after training. B: From Maren et al. (1997). Rats were given three tone-shock pairings and then given excitotoxic NMDA lesions of the $\mathrm{DH} 1,28$, or 100 days after training. After recovery, contextual freezing (peak \% time, mean \pm SEM) was assessed. A severe deficit was observed in rats that received lesions 1 day after training, but only a very mild deficit

In a second study, Maren et al. (1997) examined RA of contextual fear after excitotoxic $\mathrm{N}$-methyl-d-aspartate (NMDA) lesions of the $\mathrm{DH}$. In this study, rats received tone-shock pairings either 1 , 28 , or 100 days prior to an NMDA lesion of $\mathrm{DH}$ or sham surgery. NMDA lesions appeared somewhat larger than the electrolytic lesions used in Kim and Fanselow (1992). These excitotoxic lesions produced a near total RA of contextual fear if made 1 day after training, but those made 100 days after training produced only a mild deficit. These lesions also produced mild tone deficits. Thus, although the time-course for consolidation in this study appeared to be longer, RA was also temporally graded, as 100-day-old contextual memory was much more resistant to hippocampal damage than 1-day-old memory (Fig. 1B).

In both Kim and Fanselow (1992) and Maren et al. (1997), temporally graded RA was examined between groups, i.e., animals had only recent or remote memory. In a third study, we wanted to examine if temporally graded RA of contextual fear could be demonstrated within subjects. Within-subjects examination offers a number of advantages, including better control over performance effects and a reduction in the number of animals required. Moreover, it more closely approximates the way temporally graded RA is examined in humans. Thus, Anagnostaras et al. (1999) gave animals 10 tone-shock pairings in one context (remote memory), followed by 10 tone-shock pairings in a highly distinct context (with a different tone) 50 days later (recent memory). One day after recent training, the ani- was observed 100 days after training. C: Anagnostaras et al. (1999). Unlike A and B, temporally graded RA of contextual fear was examined within subjects in this study. Rats received 10 tone-shock pairings in one context, followed by 10 tone-shock pairings in a different context (with a different tone) $\mathbf{5 0}$ days later, followed by electrolytic DH lesions 1 day later. These rats exhibited a severe deficit of memory acquired 1 day prior to the lesion, but no significant deficit of that acquired 50 days before. Thus, these three studies show that RA of contextual fear is temporally graded after electrolytic or excitotoxic DH lesions, when examined between groups or within subjects. See original publications for additional information.

mals received electrolytic lesions of the DH (as in Kim and Fanselow, 1992) or sham surgery. In this study, DH lesions produced a severe deficit in recent contextual fear memory, reducing it by two thirds, while sparing remote contextual fear as well as remote and recent tone fear. Thus, it is apparent that unambiguous time-limited RA of contextual fear can be demonstrated within subjects (Fig. 1C).

Based on these findings, we offered a mnemonic account of contextual freezing deficits after hippocampal damage which accords well with other views of this structure's role in memory (Squire and Alvarez, 1995; O'Keefe and Nadel, 1978; Sutherland and Rudy, 1989; Squire, 1992). In this account, the hippocampus plays a role in acquiring and consolidating the unified (spatial, configural, multimodal) representation of the contextual CS (Young et al., 1994; Maren et al., 1998). The specific role we have proposed is the construction and temporary maintenance of the unified representation of the contextual CS, rather than the context-shock association or other mnemonic aspects of the fear conditioning experience. The bases for this hypothesis are discussed in detail below. Very generally, the view is that simple elements such as a tone CS or simple details that comprise a context are available to animals regardless of the integrity of the hippocampus. But animals without a hippocampus cannot establish or consolidate a memory of an integrated representation of the context as a unified whole (Fanselow, 
1980, 1990; Young et al., 1994; Maren et al., 1998; Anagnostaras et al., 1999).

This role for the DH is in contrast to that proposed for the amygdala. After NMDA lesions to the basolateral amygdala, Maren et al. (1996a) found a complete loss of contextual and tone fear even when lesions were made 28 days after training, and there is no evidence of a temporal gradient for retrograde amnesia in amygdala-dependent Pavlovian fear conditioning (Lee et al., 1996). Thus, the amygdalar complex plays a general role in contextual and tone fear for some time after acquisition. In contrast to the proposed role for the hippocampus in the acquisition and temporary maintenance of the contextual CS, the amygdala may mediate CS-US associations, the shock US representation, and perhaps even the production of fear responses (i.e., URs and CRs) as well (Maren and Fanselow, 1996; Lee et al., 1996; Campeau et al., 1992; Rogan et al., 1997).

Nonetheless, several recent controversies have emerged which apparently weaken the case for a specific and selective hippocampal role in contextual fear memory. First, several studies have found an absence of anterograde amnesia of contextual fear when hippocampal lesions are made prior to training (e.g., Maren et al., 1997; Frankland et al., 1998; Gerlai, 1998; Cho et al., 1999). Second, a dramatic, temporally stable, and nonspecific deficit in freezing has been observed after large kainate-colchicine lesions of the hippocampus (Weisend et al., 1996). Third, a performance account of freezing deficits after hippocampal damage was recently offered as an alternative to the mnemonic account (Good and Honey, 1997; McNish et al., 1997).

In this review we will address how these controversies may still be addressed by the standard mnemonic account of contextual freezing deficits. We will then offer a more detailed description of this mnemonic account.

\section{ABSENCE OF ANTEROGRADE AMNESIA OF CONTEXTUAL FEAR AFTER HIPPOCAMPAL DAMAGE}

Although there is considerable evidence of anterograde amnesia when DH lesions are made prior to conditioning (e.g., Phillips and LeDoux, 1992; Kim et al., 1993; Maren and Fanselow, 1997; Young et al., 1994; Maren et al., 1998), there is also evidence that contextual fear acquisition can sometimes be spared. For example, Maren et al. (1997) found that, after NMDA lesions of DH, retrograde amnesia was severe, but contextual fear acquisition was completely spared. Second, Phillips and LeDoux (1994) reported an absence of AA from electrolytic DH lesions after unsignaled (i.e., when no tone is used) training. Third, Frankland et al. (1998) reported only mild impairment in contextual fear after electrolytic DH lesions, and similar observations have been made after ibotenic acid lesions in mice (Cho et al., 1999). These findings are not that surprising, however, because anterograde impairments when observed were also milder than those observed when lesions were made 1 day after training (e.g., Kim and Fanselow, 1992; Kim et al., 1993; Maren et al., 1997; Anagnostaras et al., 1999; Frankland et al, 1998).

An explanation has been offered for this discrepancy between anterograde and retrograde amnesia of contextual fear, based on alternate solutions that may be adopted in contextual fear acquisition (Maren et al., 1997, 1998; Frankland et al., 1998). We have argued that fear of contextual cues may be mediated by the unified representation of the context and/or the myriad simple individual elements that make up the context (Fig. 2). By the unified representation solution (Fig. 2B), the rat first associates all static, lowcontingency (weakly predictive) contextual elements into a single representation, which is then associated with the shock. We have argued that the hippocampus, because of its role in spatial and configural learning, may be uniquely equipped to acquire this contextual CS representation (e.g., Nadel and Willner, 1980; Nadel et al., 1985). However, contextual fear conditioning is not uniquely representational in nature, because an alternative solution exists. Since the individual elements of the context are perceived by an animal even without a hippocampus, an elemental solution (Fig. 2A) is still available to lesioned animals. The individual elements in the experimental context, such as odors and noises, may come to be directly associated with shock, and these associations would not require the hippocampus.

In the intact animal both strategies would be available, while an animal with a hippocampal lesion could only use the elemental strategy. For reasons elaborated above, animals with a functioning hippocampus will favor the unified representational solution, while hippocampally impaired animals will favor the elemental solution because that is all that is available to them. Therefore, AA will be mild if the elemental solution sufficiently solves the task demands. An intact animal that learned the unified representational solution would be severely impaired if it subsequently lost the unified representation before its memory could be consolidated (i.e., retrograde amnesia would be severe).

It is important to note that the relatively weak anterograde amnesia and robust, but time-limited retrograde amnesia appear to conflict with data from amnesics indicating profound anterograde amnesia (e.g., Scoville and Milner, 1957). However, evidence presented here indicates that hippocampal animals may acquire the classically conditioned fear task in a manner that normal animals would not, using an elemental strategy. Anterograde deficits may be greatest in variants of the contextual fear task for which elemental solutions are not efficient. Frankland et al. (1998) reported evidence supporting this view. Although DH-lesioned animals were only mildly impaired in contextual fear acquisition, they were more severely impaired in contextual fear discrimination, where mice had to discriminate two similar chambers, only one of which had been paired with shock. Frankland et al. (1998) posited that this discrimination is more difficult to solve using an elemental strategy.

Nonetheless, robust deficits in contextual fear (trained according to the standard paradigm) were observed when lesions were made 1 day after training, suggesting that normal animals use their hippocampus to acquire the contextual representation (see also Maren et al., 1997). 


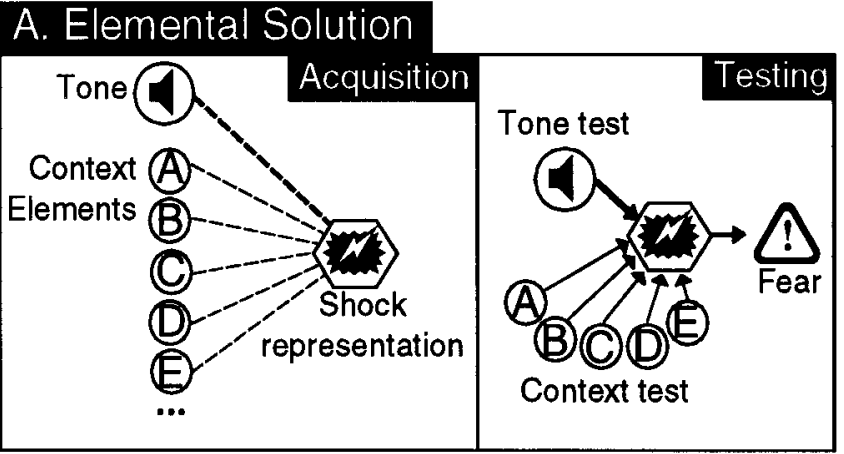

FIGURE 2. Alternate solutions that may be used in acquiring contextual fear. A: Elemental solution. As the result of pairing, the tone comes to be strongly associated with the shock representation (i.e., shock memory). Individual elements of the context are relatively poorly paired with the shock and form weak associations with the shock. During tone testing, the tone strongly arouses the shock representation, eliciting strong fear. In context testing, each individual element only weakly arouses memory of the shock, but these may summate to elicit strong fear. B: Unified representational solution. As before, the tone becomes strongly associated with shock. However, in this case, in addition to having access to the elements of the context, the hippocampus forms a unified representation of the context. This is a strong predictor of shock and forms a strong association with the

\section{TEMPORALLY STABLE AND NONSPECIFIC LOSS OF FEAR AFTER KAINATE- COLCHICINE HIPPOCAMPAL LESION}

Unpublished results of Weisend et al. (1996) indicate a temporally stable retrograde amnesia of contextual and tone freezing after kainate-colchicine hippocampal lesions. According to the authors, these lesions affect the entire hippocampus, and the data suggest that the hippocampus has a permanent role in the storage of fear memory or performance of the freezing response; studies examining hippocampal function, it was argued, should produce large, rather than restricted damage to the hippocampus. Based on these data, Nadel and Moscovitch $(1997,1998)$ concluded that spatial memories become more efficiently represented within the hippocampus over time; thus, partial lesions become less effective as the hippocampus comes to encode the memory more efficiently. However, this conclusion may need to be qualified.

First, the possibility of hippocampal lesion-induced extrahippocampal damage, particularly to the amygdala, has not been addressed. Kainic acid injection in one region is known to produce damage in distal areas that the region projects to (e.g., Mintz and Knowlton, 1993). Because we have reported a similar complete loss of context and tone conditional fear after basolateral amygdala damage (Maren et al., 1996a), exploration of this possibility is a necessity. Indeed, Jarrard $(1983,1989)$ abandoned the use of kainic acid for selective hippocampal lesions because of cell loss in areas a considerable distance from the site of injection. Substantial damage to the amygdala and cortex were reported after intrahippocampal injection of kainic acid (Jarrard and Meldrum, 1983). In mice, kainate-colchicine lesions of the hippocampus resulted in a
B. Unified Representational Solution

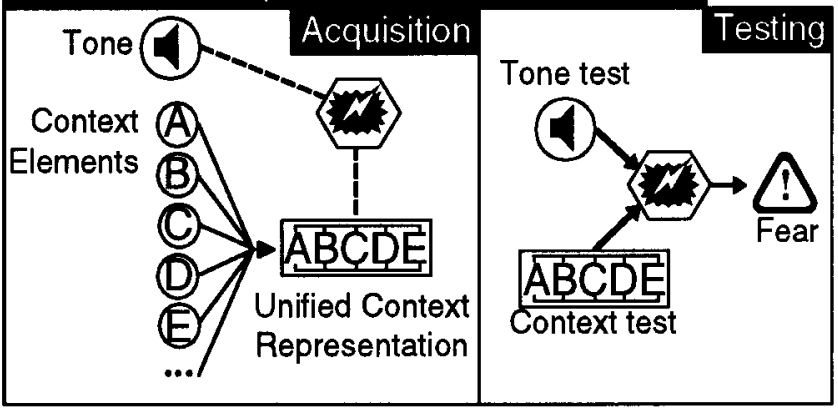

shock representation. During testing, either the tone or context can strongly arouse the shock memory and elicit strong fear. It is assumed that in normal animals both solutions are available, but the unified context representation outcompetes its individual elements because of superior predictive power and/or salience. Thus, posttraining hippocampal lesions produce a severe retrograde amnesia of contextual fear because they eliminate this unified representation that the animal associated with shock during training. However, when lesions are made prior to training, the animal may learn an elemental solution, because the unified representation is not available and therefore does not compete with the elements. Thus, during testing contextual fear may appear normal, if the task demands are such that the elemental strategy can support sufficient conditioning.

$50 \%$ shrinkage of the cortex (Logue et al., 1997). Thus, the assessment of extrahippocampal damage, particularly in the putative permanent stores for contextual fear memory (i.e., the amygdala and cortex), is critical for the interpretation of these results. It is likely that even minimal damage to the amygdala may produce significant retrograde amnesia of fear (Maren, 1998).

Second, there exists the possibility that ventral hippocampalamygdalar projections may be important for normal functioning of the amygdala. The loss of these afferents might cause significant functional disruption in the amygdala. Thus, the effects of selective ventral hippocampal and ventral angular bundle lesions (e.g., Maren and Fanselow, 1995) must be further examined before functional disruption of the amygdala can be dismissed. Indeed, Maren (1999) reported a severe and nonspecific disruption of freezing after restricted damage to the subicular region.

Third, the excitotoxic technique may be unsuitable for the localization of memory in retrograde amnesia paradigms. The massive and sustained excitatory discharge emanating from the hippocampus may be sufficient to cause "catastrophic interference" of memories already stored in other structures (e.g., McClelland et al., 1995), i.e., structures which receive afferents from the hippocampus, such as the amygdala and cortex, may try to encode this persistent and lasting noisy output. This may severely disrupt memories in these structures, perhaps especially those that were recently encoded. Thus, studies examining the effects of excitotoxic lesions of the $\mathrm{DH}$ are potentially confounded by disruption of memories stored outside the hippocampus.

This could also qualify the findings of Maren et al. (1997), in which we examined the effects of NMDA lesions of the DH. In that study, the retrograde gradient for contextual fear lasted at least 100 days, and mild tone impairments were evident. This is in 
contrast to those studies that used electrolytic lesions of the $\mathrm{DH}$, which are argued to also damage fibers of passage, but produced gradients lasting less than 50 days and no tone deficits (Kim and Fanselow, 1992; Anagnostaras et al., 1999). There seems to be a relationship between RA to remotely trained contexts, impairments in tone conditioning, and the potential for distal damage. The extension of the temporal retrograde gradient for contextual fear is accompanied by tone conditioning deficits and is produced by lesions of the kind most likely to produce distal damage and/or catastrophic interference (complete/kainate-colchicine lesions). These combined tone and remote contextual deficits are least apparent with electrolytic lesions.

Thus, substantial additional research remains before any conclusion can be reached about the effects of complete hippocampal damage on RA for contextual fear. However, the possibility remains that ventral hippocampal neurons, perhaps because of their connections with the amygdala, play a more general role in the production of the fear response than dorsal hippocampal neurons. However, temporally stable retrograde amnesia of fear after intrahippocampal kainate-colchicine injection is not good evidence for a permanent role for the hippocampus in memory storage, because this technique also likely produces disruption in the putative permanent storage sites for memory, i.e., the standard consolidation model can accommodate a temporally stable RA after this kind of damage.

PERFORMANCE ACCOUNT OF CONTEXTUAL FREEZING DEFICITS AFTER HIPPOCAMPAL LESION

Despite the specificity of the deficit we have reported for recent contextual fear (and not remote contextual fear or tone fear), a performance-based alternative to the mnemonic account of contextual freezing deficits after hippocampal lesions has been offered (Good and Honey, 1997; McNish et al., 1997; Blanchard et al., 1977). According to this view, the deficits observed in contextual freezing may be due to locomotor hyperactivity, an established effect of hippocampal lesions (Teitelbaum and Milner, 1963; Douglas and Isaacson, 1964; Maren and Fanselow, 1997; Maren et al., 1998; Anagnostaras et al., 1999; Nadel, 1968). By this account, hippocampal animals fail to exhibit normal freezing because hyperactivity caused by the lesion disrupts freezing and behavioral inhibition directly (Good and Honey, 1997; Blanchard et al., 1977; Douglas, 1967). This is a performance account, because in this view hippocampal lesions disrupt the freezing response rather than fear memory. In order to deal with the specificity of the deficit for recent contextual fear, several assumptions have been made (McNish et al., 1997). First, it is assumed that higher levels of fear are less susceptible to hippocampal lesion than low levels of fear. Second, it is assumed that remote contextual fear is stronger than recent contextual fear because of "incubation" of fear over time. Third, tone fear is assumed to be greater than contextual fear because of presumed greater CS-US contingency (McNish et al.,
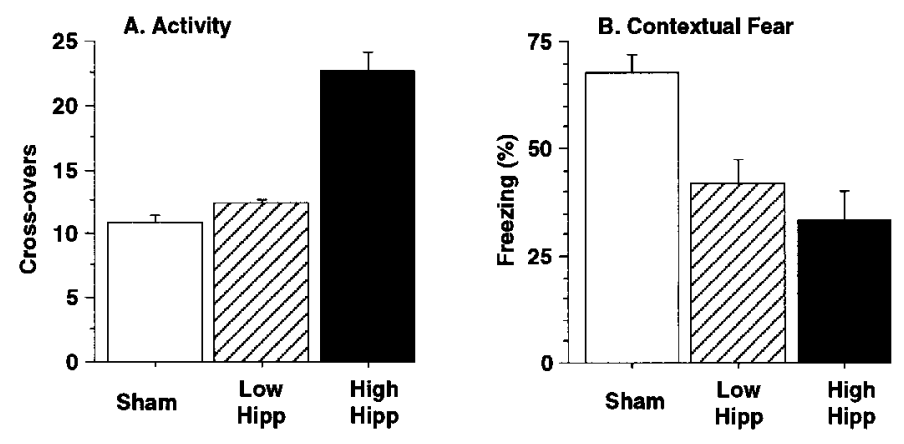

FIGURE 3. Hyperactivity and contextual freezing deficit. Data adapted from Maren et al. (1998). Hipp rats, which received electrolytic lesions of the DH prior to testing, were split into Low (below or at activity median; $n=27$ ) or High (above activity median; $n=21$ ) activity groups and compared with Sham rats $(n=49)$. A: Activity. Full cage crossovers (mean \pm SEM) were scored for the 3-min period prior to the first shock on the training day. There were significant group differences (ANOVA, F(2,94) $=47, P<0.0001$ ). High Hipp rats were significantly more active than Low Hipp (Fisher's PLSD, $P<0.0001)$ or Sham rats $(P<0.0001)$, which did not differ significantly from each other $(P>0.2)$. B: Contextual fear. The animals were returned to the conditioning chambers at least 1 day after training, and \% time freezing (mean \pm SEM) was assessed. There were significant group differences $(\mathrm{F}(2,94)=12, P<0.0001)$. High Hipp rats did not differ significantly from Low Hipp animals $(P>0.3)$, but both groups exhibited substantially less freezing than Sham rats $(P<$ 0.001 ). Thus, a robust deficit in contextual freezing can be demonstrated even in rats with DH lesions that are not significantly more active than shams. Indeed, even though High Hipp rats exhibited twice as much activity as Low Hipp or Sham animals, their freezing deficit was not much more pronounced than that of Low Hipp rats, suggesting that the contribution of hyperactivity to freezing deficit is small.

1997). We have responded to this view in detail elsewhere (Maren et al., 1998); in brief, two direct lines of evidence fail to support this view.

First, the amnesic deficit in contextual fear is unrelated to the levels of fear observed; in Kim and Fanselow (1992) and Anagnostaras et al. (1999), remote and recent contextual fear were equivalent (see Fig.1), and in Anagnostaras et al. (1999), tone fear was weaker than context fear. Because remote and recent contextual and tone fear were examined within subjects in the latter study, it is quite apparent that DH-lesioned rats can in fact freeze at the same levels as sham animals. Indeed, because of the substantial training given in all three studies, the levels of contextual fear under which retrograde amnesia of contextual fear has been reported are quite high.

Second, even when lesions are made before training, where deficits are less substantial, hyperactivity cannot predict the level of the freezing deficit (Maren et al., 1998; Maren and Fanselow, 1997). Because hyperactivity is proposed to directly disrupt freezing, according to this view, there should be a strong, negative, within-group activity-freezing correlation, and there is not. In a sample of $48 \mathrm{DH}$-lesioned rats, we found no significant correlation between hyperactivity and the contextual freezing deficit, even when hyperactivity was measured in the same conditioning chambers as freezing (Maren et al., 1998). In Figure 3, we replotted these 
data to show that hyperactivity and freezing are not directly related. In this analysis, the sample of hippocampal animals was split (median) into a group that was not significantly more active than sham controls (Low Hipp) and a group that was more than twice as active as controls (High Hipp; Fig. 3A). However, these animals did not differ in terms of amnesic severity (Fig. 3B). Thus, it appears that AA can be demonstrated even in a sample of rats that is not significantly hyperactive. Indeed, the level of freezing deficit is only slightly different in these animals from those that are over twice as active. Thus, the freezing deficit and hyperactivity do not appear to be directly related.

A number of indirect lines of evidence also fail to support the response competition view. For example, local infusion of APV, muscimol, or scopolamine into the hippocampus at the time of training selectively blocks the acquisition of contextual fear, even though the drugs are not present during testing (Young et al., 1994; Bellgowan and Helmstetter, 1995; Gale et al., 1998). Indeed, posttraining infusion of an RNA synthesis inhibitor locally into the hippocampus blocks the consolidation of contextual fear while sparing tone fear (Bailey et al., 1997).

Although there is a lack of support for the hyperactivity account of contextual freezing deficits, McNish et al. (1997) presented data showing that context-potentiated startle was not affected by $\mathrm{DH}$ lesions made immediately after training. This suggests that the deficits in contextual fear we have observed may be specific to the freezing response. Two problems in the design of their study, however, require qualification of this conclusion. First, because the measure of context-potentiated startle compared presurgery baseline and postsurgery testing, it is possible that hippocampal lesions elevated startle unconditionally, as some evidence exists that this occurs (Coover and Levine, 1972; Tilson et al., 1987; Lee and Davis, 1997). This elevation of baseline may have occluded any amnesic effect of the lesions. Second, the startle apparatus used is quite small (and under dark conditions), which may promote elemental associations. Whereas the typical chamber we use is many times larger than the rat, the startle apparatus snugly fits only the rat. Moreover, in our preparation the rats have a view of the distal features of the room, whereas in the startle apparatus used by McNish et al. (1997), the rats were in a light and sound attenuation chamber. These conditions may not be ideal for promoting the formation of a unified contextual representation, because of the poverty in complexity of the context, and the rat may instead be acquiring associations that are elemental in nature (see Maren et al., 1998). Note that even for their freezing measure, McNish et al. (1997) did not find good contextual control over freezing. In their studies where rats were trained in one context and tested in another, they still exhibited considerable freezing. With the very different and larger contexts used in our laboratory, such contextshifts between training and testing eliminate freezing (e.g., Kim and Fanselow, 1992; Maren et al., 1997; Anagnostaras et al., 1999). Thus, considerable further research is required to fully appreciate how the findings of McNish et al. (1997) will expand our understanding of the hippocampus' role in contextual fear conditioning. Moreover, additional behavioral indices of contextual fear may be useful in resolving these issues.
Nonetheless, the hyperactivity account has raised an important issue. With any measure of conditioning, any physiological manipulation (e.g., lesion, drug, mutation) has the possibility of disrupting performance of the response, rather than the learning and/or memory that mediates the behavior. This is why it is critical to include controls for such performance effects and to provide convergent lines of evidence. Hopefully, our approach with contextual fear and freezing will provide a useful example in this regard.

Thus, three controversies have complicated the view that the hippocampus has a specific role in the acquisition and storage of the contextual CS in Pavlovian fear conditioning. These controversies have substantial merit and, we believe, have actually strengthened our understanding of the hippocampus' role in contextual fear. First, an important discrepancy between the severity of anterograde and retrograde amnesia of fear after hippocampal lesions is now apparent. Second, the substantial, and perhaps nonspecific, impact of lesions of the ventral hippocampus (or extrahippocampal damage in this region) on contextual fear is becoming apparent. Third, an important performance confound, the degree to which hippocampal lesion-induced hyperactivity may have disrupted freezing, has been examined and, we believe, ruled out.

WHAT IS THE ROLE OF THE HIPPOCAMPUS IN CONTEXTUAL FREEZING?

In Pavlovian fear conditioning, contextual and tone CSs come to be associated with a shock US as the result of pairing. Based on several classic experiments, it is generally believed that the animal forms internalized neural representations of the contextual CS, tone CS, and shock US, and that conditioning results in the association of these representations. After pairing, when the animal is confronted with either the context or tone CSs, it will exhibit a fear response. It is believed that CSs elicit fear by arousing the representation (i.e., the memory) of the shock US (Rescorla, 1973, 1974), i.e., when confronted with a place or signal that was previously paired with threat, the animal remembers that these predict the threat and exhibits a defensive response that may protect it from the threat. For example, in the natural environment, freezing behavior may protect a rat from attacks by a predator in its vicinity (Fanselow and DeOca, 1998). A signal for threat (the presence of a cat, or a context associated with shock) along with freezing causes a number of adaptive responses. For example, freezing is accompanied by an opioid analgesia that reduces potential painful effects of the threat and may enhance survival (Fanselow, 1986a, 1998; Fanselow and Bolles, 1979).

Deficits in conditioning have traditionally been interpreted as deficits in one of three processes: CS processing (the CS's ability to grab the attention of associative processes), US processing (the US's ability to reinforce associative learning), or CS-US association. Whereas the processing of the tone or shock may be simple because of their unimodal and discrete nature, the processing of a contextual CS may be more complex because it is multimodal and temporally diffuse (Fanselow, 1986b, 1990). To provide signifi- 


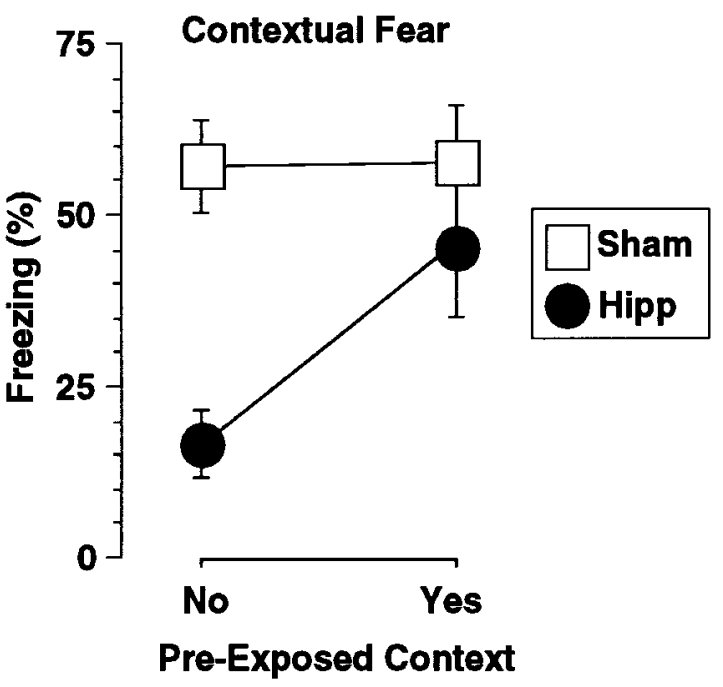

FIGURE 4. Remote context preexposure protects rats from hippocampal lesion-induced RA of recent contextual fear. Rats were preexposed to one context for $\mathbf{6 0} \mathrm{min}$ (without any shock or tone) and 35 days later were given Pavlovian fear conditioning in the same context and a highly distinct novel context. One to 2 days after conditioning, the animals received either sham or electrolytic DH lesions. Plotted here are the results of two 6-min context fear tests taken after recovery (\% time freezing, mean $\pm \mathrm{SEM})$. DH lesions produced a severe RA of fear to the not-preexposed context, but only a mild, nonsignificant impairment of fear to the preexposed context.

cant information content, the multiple elements of the contextual CS may need to be bound into a unified representation (Fig. 2B). It is specifically in the formation and consolidation of this representation that we believe the hippocampus is involved, because we have reported that remote preexposure to the context (without any shock or tone) can sometimes protect rats from anterograde amnesia (Young et al., 1994).

Recently we found that remote context preexposure can protect rats from RA after hippocampal damage as well (Fig. 4). In this study, 23 male Long-Evans rats were preexposed for $60 \mathrm{~min}$ (without shock or tone) to one context, and then 35 days later given 10 tone (10-s, 2- or 8-kHz, 85-day B/A-scale)-shock (2-s, 1-mA) pairings in the same context and a highly distinct, novel context (1 day apart; order counterbalanced; for a complete description of the procedures, see Anagnostaras et al., 1999). On the next day, they received sham $(n=12)$ or electrolytic DH lesions $(n=11)$. After a 10-day recovery period, they were given two 6-min remote and recent context freezing tests (on separate days) and two tone tests in a novel context (on separate days). The results of the context tests are depicted in Figure 4. There was a group $\times$ preexposure interaction $(\mathrm{F}(1,21)=7.7, P=0.01)$. Although RA of the not-preexposed context was severe $(\mathrm{F}(1,21)=22, P<0.0001)$, there was no significant deficit to the remotely preexposed context $(\mathrm{F}(1,21)=$ $0.8, P>0.3)$, even though both had been conditioned 1-2 days before the lesion. Moreover, fear of the not-preexposed and preexposed contexts differed for $\mathrm{DH}(\mathrm{F}(1,10)=11, P<0.01)$ but not sham rats $(\mathrm{F}(1,11)<0.1, P>0.9)$. The rats also did not exhibit deficits to either of the two tones used during conditioning (data not shown; group $\times$ context preexposure interaction, $\mathrm{F}(1,21)=$
$0.8, P>0.3$; main effect of lesion, $\mathrm{F}(1,21)<0.1, P>0.9)$. Thus, remote context preexposure appears to be sufficient to protect rats from the otherwise devastating RA of recent contextual fear that DH lesions produce.

Hippocampal LTP may be required for assembling these stimulus elements into a cognitive representation of the context as well, because infusion of the NMDA receptor antagonist D, L-2-amino5-phosphonovaleric acid (APV) into the hippocampus is sufficient to block the acquisition of contextual fear (Young et al., 1994).

There is evidence that the other mnemonic processes (shock representation and CS-US association) required for fear conditioning are supported by the amygdala (Maren and Fanselow, 1996; Rogan et al., 1997; Campeau et al., 1992; Fanselow, 1998). For example, local infusion of APV into the basolateral amygdala blocks the acquisition of tone or contextual fear (Fanselow and Kim, 1994; Maren et al., 1996b; Campeau et al., 1992), and lesions of this region produce a severe anterograde and temporally stable retrograde amnesia (Maren et al., 1996a; Lee et al., 1996). Thus, the amygdala supports the association of the unified contextual representation (acquired in the hippocampus) with the shock US in a way similar to which it supports tone-shock associations. Context-shock association may be via hippocampal-amygdala LTP, as high-frequency stimulation of the pathways carrying information from the hippocampus to the amygdala produces LTP, and lesions of these projections selectively block the acquisition of contextual fear (Maren and Fanselow, 1995; Maren, 1996).

It also appears that, in normal animals, the hippocampal unified representational solution actively competes with an alternative elemental solution. Whereas pretraining lesions produce only a mild impairment in contextual conditioning, posttraining lesions are devastating. This suggests that even though animals with hippocampal lesions can use elemental strategies to acquire contextual fear, intact animals do not. This may result from the unified representation competing with multiple elemental representations for association with the shock.

Competition between cues is such a ubiquitous finding in learning experiments that it is at the core of many models of conditioning (Sutherland and Mackintosh, 1971; Rescorla and Wagner, 1972; Wagner and Brandon, 1989). There are two factors that favor a particular stimulus in this competition for associative strength. One is that better predictors win over poorer predictors. The other is that more salient stimuli win over weaker stimuli. Both of these factors should favor the unified representation over the elemental stimuli. Because a rat could only perceive a subset of elements of the context at any instant, the unitary representation will be more reliably paired with shock than any constituent element and thus may have superior predictive power. For example, a cue that is paired with shock on $50 \%$ of its trials will condition strongly if it is the best predictor available, but not at all if a better predictor is available (Wagner et al., 1968). Additionally, it seems likely that any single element of the context will be less salient than the unified representation of these elements. Another well-established finding in the literature, i.e., overshadowing, speaks to this issue. In overshadowing, a weak stimulus conditions well on its own, but poorly if presented in compound with a more salient stimulus (Pavlov, 1927; Mackintosh, 1975). When the hippocam- 
pus is intact, the unified representation may overshadow the single elements. Note that learning theories explain the overshadowing and the predictive validity effects with the same algorithm (e.g., Rescorla and Wagner, 1972). Therefore, a fundamental assumption from theories of Pavlovian conditioning is that if there are both elements of a context (this would have to be true) and the elements can be unified into a contextual representation, the elements and representation must compete. Fortunately, this a priori prediction from Pavlovian theory also explains a number of subtleties of the effects of hippocampal lesions on contextual fear conditioning.

For example, Phillips and LeDoux (1994) reported that hippocampal lesions produce AA of contextual fear when initial training is signaled (with a tone) but not when it is unsignaled (without a tone). When a tone (that has high salience and predictive power) signals shock, it would compete for association with the shock with the unified contextual representation and the elemental stimuli. While we have argued that hippocampal-lesioned animals can sometimes acquire contextual fear by elemental associations, the addition of a competing cue, the tone, which is both more salient and a better predictor, could weaken elemental contextual associations enough to reveal a deficit. However, the relative lack of competition in normal animals between the context representation and tone signal suggests that they have similar salience and predictive power.

With regard to the processes of consolidation, the study of contextual fear may offer important insights. For example, in one view, recent memory traces (engrams) actually migrate from the hippocampus to distal locations such as the cortex over the consolidation gradient (months to years). In another view, engrams are established initially at "slow-learning" permanent locations, and the hippocampus only plays a role in temporarily keeping these engrams stable (e.g., McClelland et al., 1995). We believe that studies of contextual fear favor the latter (for a historical discussion of these views, see Squire and Alvarez, 1995). For example, if the recently acquired unified contextual CS (located in the hippocampus) migrated during consolidation to another location, it is unclear how it would maintain its associative connection with the US representation (located in the amygdala). This would not be a problem if the hippocampus was only involved in temporarily stabilizing a nonmigratory trace elsewhere. These speculations remain to be tested, of course, but contextual fear may offer an efficient means to examine the processes underlying consolidation.

The assembly of the unified contextual CS is similar to the role the hippocampus is argued to play in many paradigms. One important advantage is that because a functional hippocampus is not required for the performance of contextual freezing, time-limited retrograde amnesia, such as is observed in human declarative memory, can readily be demonstrated in context fear. This is in contrast to the spatial Morris water and Olton radial maze tasks, in which online hippocampal processing seems to be required for high levels of performance, perhaps because of navigational or working memory demands (Knowlton and Fanselow, 1998). One weakness of contextual fear in examining the anterograde influence on hippocampal function is that because acquisition of contextual fear can sometimes appear normal even in animals with hippocampal lesions, evidence of acquisition of contextual fear cannot be taken as unequivocal evidence that hippocampal function is normal (Gerlai, 1998). This is in contrast to maze learning tasks, which apparently show more robust anterograde amnesia. While RA for contextual fear provides clear evidence, we need to refine the anterograde amnesia tests in a way to selectively control the unified vs. elemental solutions. Nonetheless, taken together, evidence from contextual freezing and maze learning experiments, which can often be done in the same animals, may be a good indicator of functional disruption of the same neural system that mediates human declarative memory.

\section{Acknowledgments}

We thank Paul Frankland and Jennifer Sage for their thoughtful comments on an earlier version of this manuscript. This research was supported by NSF grant (IBN 9723295) to M.S.F. S.G.A. was supported by a UCLA C.M. Kernan Dissertation Year Fellowship.

\section{REFERENCES}

Anagnostaras SG, Maren S, Fanselow MS. 1999. Temporally graded retrograde amnesia of contextual fear after hippocampal damage in rats: within-subjects examination. J Neurosci 19:1106-1114.

Bailey DJ, Sun W, Kim JJ, Helmstetter FJ. 1997. Inhibition of RNA synthesis in the amygdala and hippocampus selectively blocks acquisition of Pavlovian fear conditioning. Soc Neurosci Abstr 23:1609.

Bellgowan PSF, Helmstetter FJ. 1995. Effects of muscimol applied to the dorsal hippocampus on the acquisition and expression of cued versus contextual fear conditioning. Soc Neurosci Abstr 21:1219.

Blanchard DC, Blanchard RJ, Lee MC, Fukunaga KK. 1977. Movement arrest and the hippocampus. Physiol Psychol 5:331-335.

Bolhuis JJ, Stewart CA, Forrest EM. 1994. Retrograde amnesia and memory reactivation in rats with ibotenate lesions to the hippocampus or subiculum. Q J Exp Psychol [B] 47:129-150.

Bolles RC. 1970. Specifies-specific defense reactions and avoidance learning. Psychol Rev 77:32-48.

Campeau S, Miserendino MJ, Davis M. 1992. Intra-amygdala infusion of the N-methyl-D-aspartate receptor antagonist AP5 blocks acquisition but not expression of fear-potentiated startle to an auditory conditioned stimulus. Behav Neurosci 106:569-174.

Cho YH, Kesner RP. 1996. Involvement of entorhinal cortex or parietal cortex in long-term spatial discrimination memory in rats: retrograde amnesia. Behav Neurosci 110:436-442.

Cho YH, Beracochea D, Jaffard R. 1993. Extended temporal gradient for the retrograde and anterograde amnesia produced by ibotenate entorhinal cortex lesions in mice. J Neurosci 13:1759-1766.

Cho YH, Friedman E, Silva AJ. 1999. Ibotenate lesions of the hippocampus impair spatial learning but not contextual fear conditioning in mice. Behav Brain Res 98:77-87.

Coover GD, Levine S. 1972. Auditory startle response of hippocampectomized rats. Physiol Behav 9:75-77.

Douglas RJ. 1967. The hippocampus and behavior. Psychol Bull 67, 416-442.

Douglas RJ, Isaacson RL. 1964. Hippocampal lesions and activity. Psychonomic Sci 1:187-188.

Fanselow MS. 1980. Conditioned and unconditional components of post-shock freezing. Pavlov J Biol Sci 15:177-182. 
Fanselow MS. 1986a. Conditioned fear-induced opiate analgesia: a competing motivational state theory of stress-analgesia. Ann NY Acad Sci 467:40-54.

Fanselow MS. 1986b. Associative vs. topographical accounts of the immediate shock-freezing deficit in rats: implications for the response selection rules governing species-specific defense reactions. Learn Motiv 17:16-39.

Fanselow MS. 1990. Factors governing one-trial contextual conditioning. Anim Learn Behav 18:264-270.

Fanselow MS. 1998. Pavlovian conditioning, negative feedback, and blocking: mechanisms that regulate association formation. Neuron 20:625-627.

Fanselow MS, Bolles RC. 1979. Naloxone and shock-elicited freezing in the rat. J Comp Physiol Psychol 93:736-744.

Fanselow MS, DeOca BM. 1998. Defensive behaviors. In: Greenberg G, Haraway MM, editors. Comparative psychology: a handbook. New York: Garland Publishing. p 653-655.

Fanselow MS, Kim JJ. 1994. Acquisition of contextual Pavlovian conditioning is blocked by application of an NMDA receptor antagonist D,L-2-amino-5-phosphonovaleric acid to the basolateral amygdala. Behav Neurosci 108:210-220.

Frankland PW, Cestari V, Filipkowski RK, McDonald RJ, Silva AJ. 1998. The dorsal hippocampus is essential for context discrimination but not for contextual conditioning. Behav Neurosci 112:863-874.

Gale GD, Anagnostaras SG, Fanselow MS. 1998. Cholinergic modulation of Pavlovian fear conditioning: selective disruption of contextual conditioning by intra-hippocampal infusion of scopolamine. Soc Neurosci Abstr 24:1904.

Gerlai R. 1998. Contextual learning and cue association in fear conditioning in mice: a strain comparison and a lesion study. Behav Brain Res 95:191-201.

Good M, Honey RC. 1997. Dissociable effects of selective lesions to hippocampal subsystems on exploratory behavior, contextual learning, and spatial learning. Behav Neurosci 111:487-493.

Jarrard LE. 1983. Selective hippocampal lesions and behavior: effects of kainic acid lesions on performance of place and cue tasks. Behav Neurosci 97:873-889.

Jarrard LE. 1989. On the use of ibotenic acid to lesion selectively different components of the hippocampal formation. J Neurosci Methods 29: 251-259.

Jarrard LE, Meldrum BS. 1990. Neurotoxicity of intrahippocampal injections of excitatory amino acids: protective effects of CPP. Soc Neurosci Abstr 16:429.

Kim JJ, Fanselow MS. 1992. Modality-specific retrograde amnesia of fear. Science 256:675-677.

Kim JJ, Rison RA, Fanselow MS. 1993. Effects of amygdala, hippocampus, and periaqueductal gray lesions on short- and long-term contextual fear. Behav Neurosci 107:1093-1098.

Kim JJ, Clark RE, Thompson RF. 1995. Hippocampectomy impairs the memory of recently, but not remotely, acquired trace eyeblink conditioned responses. Behav Neurosci 109:195-203.

Knowlton BJ, Fanselow MS. 1998. The hippocampus, consolidation, and on-line memory. Curr Opin Neurobiol 8:293-296.

Lee Y, Davis M. 1997. Role of the septum in the excitatory effect of corticotropin-releasing hormone on the acoustic startle reflex. J Neurosci 17:6424-6433.

Lee Y, Walker D, Davis M. 1996. Lack of temporal gradient of retrograde amnesia following NMDA-induced lesions of the basolateral amygdala assessed with the fear-potentiated startle paradigm. Behav Neurosci 110:836-839.

Logue SF, Paylor R, Wehner JM. 1997. Hippocampal lesions cause learning deficits in inbred mice in the Morris water maze and conditionedfear task. Behav Neurosci 111:104-113.

Mackintosh NJ. 1975. A theory of attention: variations in the associability of stimuli with reinforcement. Psychol Rev 82:276-298.
Maren S. 1996. Synaptic transmission and plasticity in the amygdala. An emerging physiology of fear conditioning circuits. Mol Neurobiol 13: $1-22$.

Maren S. 1998. Overtraining does not mitigate contextual fear conditioning deficits produced by neurotoxic lesions of the basolateral amygdala. J Neurosci 18:3088-3097.

Maren S. 1999. Neurotoxic or electrolytic lesions of the ventral subiculum produce deficits in acquisition and expression of fear conditioning in rats. Behav Neurosci 113:289-289.

Maren S, Fanselow MS. 1995. Synaptic plasticity in the basolateral amygdala induced by hippocampal formation stimulation in vivo. J Neurosci $15: 7548-7564$.

Maren S, Fanselow MS. 1996. The amygdala and fear conditioning: has the nut been cracked? Neuron 16:237-240.

Maren S, Fanselow MS. 1997. Electrolytic lesions of the dorsal hippocampus, fimbria-fornix, or entorhinal cortex produce anterograde deficits in contextual fear conditioning in rats. Neurobiol Learn Mem 67:142149 .

Maren S, Aharonov G, Fanselow MS. 1996a. Retrograde abolition of conditional fear after excitotoxic lesions in the basolateral amygdala of rats: absence of a temporal gradient. Behav Neurosci 110:718-726.

Maren S, Aharonov G, Stote DL, Fanselow MS. 1996b. N-methyl daysaspartate receptors in the basolateral amygdala are required for both acquisition and expression of conditional fear in rats. Behav Neurosci 110:1365-1374.

Maren S, Aharonov G, Fanselow MS. 1997. Neurotoxic lesions of the dorsal hippocampus and Pavlovian fear conditioning in rats. Behav Brain Res 88:261-274.

Maren S, Anagnostaras SG, Fanselow MS. 1998. The startled seahorse: is the hippocampus necessary for contextual fear conditioning? Trends Cogn Sci 2:39-42.

McClelland JL, McNaughton BL, O'Reilly RC. 1995. Why there are complementary learning systems in the hippocampus and neocortex: insights from the successes and failures of connectionist models of learning and memory. Psychol Rev 102:419-437.

McNish KA, Gewirtz JC, Davis M. 1997. Evidence of contextual fear after lesions of the hippocampus: a disruption of freezing but not fearpotentiated startle. J Neurosci 17:9353-9360.

Milner B, Squire LR, Kandel ER. 1998. Cognitive neuroscience and the study of memory. Neuron 20:445-468.

Mintz M, Knowlton BJ. 1993. Dissociation of kainic acid lesion effects on the asymmetry of rotation and lateral head movements. Brain Res Bull 31:641-647.

Morris RGM. 1983. An attempt to dissociate "spatial mapping" and "working memory" theories of hippocampal function. In: Seifert W, editor. The neurobiology of the hippocampus. London: Academic Press. p 405-432.

Nadel L. 1968. Dorsal and ventral hippocampus lesions and behavior. Physiol Behav 3:891-900.

Nadel L, Moscovitch M. 1997. Memory consolidation, retrograde amnesia and the hippocampal complex. Curr Opin Neurobiol 7:217-227.

Nadel L, Moscovitch M. 1998. Hippocampal contributions to cortical plasticity. Neuropharmacology 37:431-439.

Nadel L, Willner J. 1980. Context and conditioning: a place for space. Physiol Psychol 8:218-228.

Nadel L, Willner J, Kurz EM. 1985. Cognitive maps and environmental context. In: Balsam PD, Tomie A, editors. Context and learning. London: Erlbaum. p 285-406.

O’Keefe J, Nadel L. 1978. The hippocampus as a cognitive map. Oxford: Clarendon Press.

Olton DS, Becker JT, Handelmann GE. 1979. The hippocampus, space, and memory. Behav Brain Sci 2:313-365.

Pavlov IP. 1927. Conditioned reflexes. Anrep GV, translator. London: Oxford University Press.

Phillips RG, LeDoux JE. 1992. Differential contribution of amygdala and hippocampus to cued and contextual fear conditioning. Behav Neurosci 106:274-285. 
Phillips RG, LeDoux JE. 1994. Lesions of the dorsal hippocampal formation interfere with background but not foreground contextual fear conditioning. Learn Mem 1:34-44.

Reed JM, Squire LR. 1998. Retrograde amnesia of facts and events: findings from four new cases. J Neurosci 18:3943-3954.

Rempel-Clower NL, Zola SM, Squire LR, Amaral DG. 1996. Three cases of enduring memory impairment after bilateral damage limited to the hippocampal formation. J Neurosci 15:5233-5255.

Rescorla RA. 1973. Effect of US habituation following conditioning. J Comp Physiol Psychol 82:137-143.

Rescorla RA. 1974. Effect of inflation on the unconditioned stimulus value following conditioning. J Comp Physiol Psychol 86:101-106.

Rescorla RA, Wagner AR. 1972. A theory of Pavlovian conditioning: Variations in the effectiveness of reinforcement and nonreinforcement. In classical conditioning II: current theory and research. In: Black AH, Prokasy WF, editors. New York: Appleton Century Crofts. p 65-99.

Rogan MT, Staubli UV, LeDoux JE. 1997. Fear conditioning induces associative long-term potentiation in the amygdala. Nature 390:604-607.

Scoville WB, Milner B. 1957. Loss of recent memory after bilateral hippocampal lesions. J Neurol Neurosurg Psychiatry 20:11-21.

Squire LR. 1992. Memory and the hippocampus: a synthesis from findings with rats, monkeys, and humans. Psychol Rev 99:195-231.

Squire LR, Alvarez P. 1995. Retrograde amnesia and memory consolidation: a neurobiological perspective. Curr Opin Neurobiol 5:178-183.

Sutherland NS, Mackintosh MJ. 1971. Mechanisms of animal discrimination learning. New York: Academic Press.

Sutherland RJ, Rudy RJ. 1989. Configural association theory: the role of the hippocampal formation in learning, memory, and amnesia. Psychobiology 17:129-144.
Teitelbaum H, Milner P. 1963. Activity changes following partial hippocampal lesions in rats. J Comp Physiol Psychol 56:281-289.

Tilson HA, Rogers BC, Grimes L, Harry GJ, Peterson NJ, Hong JS, Dyer, RS. 1987. Time daysependent neurobiological effects of colchicine administered directly into the hippocampus of rats. Brain Res 408: 163-172.

Wagner AR, Brandon SE. 1989. Evolution of a structured connectionist model of Pavlovian conditioning (AESOP). In: Klein SB, Mowrer RR, editors. Contemporary learning theories: Pavlovian conditioning and the status of learning theory. Hillsdale, NJ: Erlbaum. p 149-189.

Wagner AR, Logan FA, Haberlandt K, Price T. 1968. Stimulus selection in animal discrimination learning. J Exp Psychol 76:171-180.

Weisend MP, Astor RS, Sutherland RJ. 1996. The specificity and temporal characteristics of retrograde amnesia after hippocampal lesions. Soc Neurosci Abstr 22:1118.

Wiig KA, Cooper LN, Bear MF. 1996. Temporally graded retrograde amnesia following separate and combined lesions of the perirhinal cortex and fornix in the rat. Learn Mem 3:313-325.

Winocur G. 1990. Anterograde and retrograde amnesia in rats with dorsal hippocampal or dorsomedial thalamic lesions. Behav Brain Res 38: 145-154.

Young SL, Bohenek DL, Fanselow MS. 1994. NMDA processes mediate anterograde amnesia of contextual fear conditioning induced by hippocampal damage: immunization against amnesia by context preexposure. Behav Neurosci 108:19-29.

Zola-Morgan SM, Squire LR. 1990. The primate hippocampal formation: evidence for a time-limited role in memory storage. Science 250: 288-290. 